\title{
Substituição do iodo por fitoterápicos no tratamento do coto umbilical de cabritos
}

\author{
Silva, T.G.P. ${ }^{1}$; Silva, J.B.C. ${ }^{\text {; }}$ Guim, A. ${ }^{\text {; }}$ Silva, J.L. ${ }^{2}$; Maciel, M.V. ${ }^{1}$ e Ferreira, M.P.B. ${ }^{1}$
}

'Departamento de Zootecnia. Universidade Federal Rural de Pernambuco. UFRPE. Recife. Pernambuco. Brasil. ${ }^{2}$ Centro Multidisciplinar de Barra, Universidade Federal do Oeste da Bahia. UFOB. Barra. Bahia. Brasil.

\section{PalaVRas ChaVe ADICIONAIS}

Avaliação econômica.

Infecções neonatais.

Ruminantes.

Tempo de cicatrizaç̃óo.

\section{RESUMO}

Objetivou-se avaliar o tempo de cicatrização e de queda do coto umbilical de caprinos recém-nascidos, a partir da utilização de fitoterápicos de uso tópico à base de aroeira (Schinus terebinthifolius Raddi) ou babosa (Aloe barbadensis Miller) em substituição ao iodo comercial. Analisou-se também o emprego de diferentes metodologias de aplicação dos fitoterápicos e a viabilidade econômica da utilização desses produtos. No experimento I foram utilizados 28 cabritos neonatos da raça Saanen, sendo sete animais em cada tratamento, com peso médio ao nascimento de $2,69 \mathrm{~kg}$. No experimento II foram utilizados 15 cabritos neonatos da raça Saanen, com peso médio ao nascimento de $2,66 \mathrm{~kg}$. Os animais foram distribuídos em um delineamento inteiramente casualizado, com três tratamentos e cinco repetições. Os tratamentos experimentais consistiram em: a) grupo controle - tratamento do coto umbilical em solução de iodo a $10 \%$; b) grupo 1 - tratamento do coto umbilical com tintura de aroeira; e c) grupo 2 - tratamento do coto umbilical com tintura de babosa. Verificou-se que o tempo médio para cicatrização e queda do coto umbilical não diferiu entre os animais submetidos aos tratamentos aroeira e babosa (20,8 e 18,6 dias), com uma aplicação diária por três dias consecutivos, comparado ao grupo controle $(20,4$ dias). As tinturas fitoterápicas à base de aroeira ou babosa podem substituir a tintura de iodo a $10 \%$ e demonstram maior viabilidade econômica para utilização no processo de cicatrização e queda do coto umbilical de cabritos.

\section{Replacement of iodine by phytotherapics on the treatment of umbilical cord stump of goat kids}

\section{SUMMARY}

The aim of this study was to evaluate the healing time and downfall of umbilical stump of newborn goat kids, from the topical use of phytotherapics-based of aroeira (Schinus terebinthifolius Raddi) or babosa (Aloe barbadensis Miller) replacing the commercial iodine. Also it was evaluated the use of different methodologies of phytotherapics application and economic feasibility of using these products. In the experiment I were used 28 neonates Saanen goat kids, seven animals in each treatment, with an average birth weight of $2.69 \mathrm{~kg}$. In the experiment II were used 15 neonates Saanen goat kids with an average birth weight of $2.66 \mathrm{~kg}$. The animals were distributed in a completely randomized design with three treatments and five replications. The treatments consisted of: a) control group - umbilical stump treatment with iodine solution 10\%; b) group 1 - umbilical stump treatment with aroeira's tincture; and c) Group 2 - umbilical stump treatment with babosa's extract. It was found that the average healing time and downfall of umbilical stump did not differ between animals submitted to the aroeira and babosa treatments (20.8 and 18.6 days), with a daily application for three consecutive days, compared to the control group (20.4 days). The phytotherapics tinctures based on aroeira or babosa can replace the iodine tincture to $10 \%$, demonstrating greater economic viability for using in the healing process and downfall of umbilical stump of goat kids.

\section{Cronología del artículo.}

Recibido/Received: 23.01.2017

Aceptado/Accepted: 04.04.2018

On-line: 15.04 .2018

Correspondencia a los autores/Contact e-mail:

silva_janainalima@yahoo.com.br

\section{INTRODUÇÃO}

O Brasil apresenta elevada diversidade de produtos naturais que são utilizados para a prevenção ou tratamento de doenças, tanto para uso humano quanto animal. As plantas medicinais possuem substâncias bioativas (compostos químicos), produzidas durante seu metabolismo, que lhes conferem ação terapêutica, podendo ser utilizadas como tratamento alternativo ou complementar na produção animal. No entanto, a utilização de produtos naturais na criação animal ainda é pouco explorada (Catalan et al. 2012, p. 15).

Dentre as plantas com potencial para elaboração de fitoterápicos pode-se citar a aroeira e a babosa (Men- 
donça et al. 2014, p. 3). A aroeira (Schinus terebinthifolius Raddi), da família Anacardiaceae, é comumente encontrada na vegetação litorânea do nordeste brasileiro, sendo sua casca amplamente utilizada pela população como anti-inflamatório e cicatrizante (Castelo Branco Neto et al. 2006, p. 2). Estudo com espécies do gênero Schinus descreve a ocorrência de terpenoides, ácidos graxos e flavonoides em Schinus terebinthifolius (Moneam e Ghoneim 1986, p. 395).

A Aloe vera, também conhecida como Aloe barbadensis Miller (família Liliaceae) ou popularmente como babosa, há muito tempo é utilizada como medicamento. A partir do processamento de suas folhas obtém-se um exsudato amargo e um gel mucilaginoso. $\mathrm{O}$ exsudato é considerado pela literatura como a droga "aloe", líquido de coloração amarelo-avermelhada, rico em compostos antracênicos. O gel mucilaginoso de aspecto incolor é proveniente do parênquima da folha e tem sido utilizado no tratamento de queimaduras, ulcerações da pele e cicatrização de feridas (Maia-Filho et al. 2011, p. 147).

O uso de plantas medicinais para o tratamento de animais tem aumentado progressivamente, principalmente devido à pressão do consumidor, que cada vez mais busca por produtos obtidos de forma ecologicamente correta (Oliveira et al. 2009, p. 2). O uso frequente dos quimioterápicos propicia o desenvolvimento de resistência aos produtos, além de promover danos aos animais parasitados e ao homem, que consome os produtos de origem animal (Olivo et al. 2008, p. 406). Nesse sentido, vários fitoterápicos usados na medicina popular têm sido testados na cicatrização de feridas cutâneas (Castelo Branco Neto et al. 2006, p. 2) e prevenção de mastite (Amaro et al. 2011, p. 8). No entanto, são escassos os estudos com produtos fitoterápicos para tratamento do coto umbilical de animais neonatos.

Durante o parto ocorre a ruptura do cordão umbilical e a perda de sua função, ficando o coto umbilical exposto a agentes patogênicos, o que pode provocar inúmeras doenças no recém-nascido, redução no desempenho e até a morte do animal. Segundo Medeiros et al. (2005, p. 202), na maioria das propriedades há ausência do tratamento do coto umbilical ou realização deste de forma inadequada, seja com utilização de iodo ou de repelentes, o que aliado à má nutrição das crias, cujas mães são utilizadas para produção de leite, levam os animais a condições de estresse, predispondo às infecções neonatais.

Diante do exposto, objetivou-se avaliar o tempo de cicatrização e de queda do coto umbilical de caprinos recém-nascidos, a partir da utilização de fitoterápicos de uso tópico à base de aroeira ou babosa em substituição ao iodo comercial. Além disso, analisou-se o emprego de diferentes metodologias de aplicação dos fitoterápicos e a viabilidade econômica da utilização desses produtos.

\section{MATERIAIS E MÉTODOS}

Foram realizados dois experimentos no Setor de Caprinos e Ovinos do Departamento de Zootecnia da
Universidade Federal Rural de Pernambuco (UFRPE), Recife, Pernambuco, sob as coordenadas geográficas: $8^{\circ} 04^{\prime} 03^{\prime \prime} \mathrm{S}$ e $34^{\circ} 55^{\prime} 00^{\prime \prime} \mathrm{W}$, com altitude de 4 metros.

Após o nascimento, os animais de ambos os estudos foram pesados em balança digital, identificados com colar, procedendo-se o corte do umbigo com uma tesoura esterilizada a uma distância de quatro centímetros do ventre do animal. Logo após, realizou-se a imersão do coto umbilical por um minuto no recipiente contendo $25 \mathrm{~mL}$ do produto correspondente a cada tratamento experimental.

As plantas medicinais utilizadas na produção das tinturas fitoterápicas e do extrato foram: aroeira (Schinus terebinthifolius Raddi) e babosa (Aloe barbadensis Miller). A aroeira foi proveniente do próprio local de estudo, devido à ocorrência de exemplares desta árvore. A babosa foi obtida de canteiros da comunidade circunvizinha ao Campus da Universidade.

A tintura de aroeira foi produzida utilizando-se entrecascas da planta e aguardente de cana-de-açúcar $\left(40^{\circ}\right.$ GL), em uma proporção de $200 \mathrm{~g}$ de entrecascas para cada $800 \mathrm{~mL}$ da aguardente. $\mathrm{O}$ extrato de babosa foi obtido triturando-se a babosa em liquidificador comercial, sem adição de água e com utilização imediata após sua fabricação. A tintura de babosa foi confeccionada a partir das folhas e álcool etílico hidratado ( $70^{\circ}$ INPM), misturando-se em liquidificador $200 \mathrm{~g}$ do gel extraído das folhas e $800 \mathrm{~mL}$ de álcool. Após a fabricação, as tinturas foram armazenadas por 20 dias em recipientes de vidro, de cor escura a temperatura ambiente, sem contato direto com luz solar. A tintura de iodo a 10\% (tratamento controle) foi adquirida no comércio.

No experimento I (ensaio preliminar) foram utilizados 28 cabritos neonatos da raça Saanen, sendo sete animais em cada tratamento, com peso médio ao nascimento de $2,69 \mathrm{~kg}$. Os tratamentos experimentais consistiram em: a) grupo controle (GC) - tratamento convencional do coto umbilical (imersão do coto umbilical em solução de iodo a $10 \%$, duas vezes ao dia por três dias consecutivos); b) grupo 1 (G1) - tratamento do coto umbilical com tintura de iodo a $10 \%$; c) grupo 2 (G2) - tratamento do coto umbilical com tintura de aroeira; e d) grupo 3 (G3) - tratamento do coto umbilical com aplicação tópica de extrato de babosa. Nos cabritos dos grupos 1, 2 e 3, o tratamento foi realizado a cada 12 horas até a completa cicatrização e queda do coto umbilical.

A partir da análise dos resultados do ensaio preliminar percebeu-se a ineficácia da metodologia de aplicação dos produtos (a cada 12 horas até a completa cicatrização e queda do coto umbilical), sendo então realizado o experimento de campo, com redução do número de aplicações, comparando-se as tinturas fitoterápicas e a solução comercial de iodo utilizadas, seguindo-se a recomendação da metodologia tradicional, que consiste no tratamento do coto umbilical na frequência de duas vezes ao dia por três dias consecutivos.

No experimento II, foram utilizados 15 cabritos neonatos da raça Saanen, com peso médio ao nascimento de 2,66 kg. Os animais foram distribuídos em 
um delineamento inteiramente casualizado, com três tratamentos e cinco repetições. Os tratamentos experimentais consistiram em: a) grupo controle (GC) - tratamento do coto umbilical em solução de iodo a 10\%; b) grupo 1 (G1) - tratamento do coto umbilical com tintura de aroeira; e c) grupo 2 (G2) - tratamento do coto umbilical com tintura de babosa. As aplicações de todas as tinturas, inclusive a de iodo, foram realizadas uma vez ao dia durante três dias consecutivos, com avaliação clínica diária até a completa cicatrização e queda do coto umbilical.

Em ambos os experimentos, os animais foram alimentados naturalmente com colostro materno. A partir do terceiro dia de vida, quando separados das matrizes, iniciou-se o aleitamento artificial, por mamadeiras individuais, duas vezes ao dia ( $8 \mathrm{~h} 00$ e 16h00) em quantidade equivalente a $20 \%$ do peso corporal (PC). Na segunda semana após o nascimento foi introduzida alimentação sólida, fornecida duas vezes ao dia (08h00 e 16h00), sendo o volumoso à base de feno de capim tifton-85 (Cynodon spp.) e o concentrado formulado com farelo de milho $(58 \%)$, farelo de soja $(24 \%)$, farelo de trigo $(18 \%)$ e sal mineral, em quantidade equivalente a $4 \%$ do PC, além de água à vontade.

Realizou-se ainda a análise econômica da utilização das tinturas fitoterápicas. Os dados do ensaio preliminar foram submetidos à análise estatística descritiva, com determinação da média, utilizando-se o programa Microsoft Office Excel ${ }^{\circledR}$ Versão 2011. No experimento II, os dados foram submetidos à análise de variância e as médias comparadas pelo teste de Tukey, ao nível de significância de 5\%.

\section{RESULTADOS E DISCUSSÃO}

No ensaio preliminar verificou-se que o tempo médio de cicatrização e queda do coto umbilical nos cabritos pertencentes aos grupos 1, 2 e 3 (31, 40 e 42 dias, respectivamente) foi superior aos animais do grupo controle (11 dias), mesmo quando o produto utilizado foi a tintura de iodo. De acordo com a ACCOMIG (n.d.), o uso de tintura de iodo a $10 \%$ promove a cicatrização e queda do coto umbilical entre o sétimo e o décimo quinto dia após o tratamento, o que foi confirmado apenas pelos animais do grupo controle (GC).

Observou-se que o maior número de aplicações das tinturas e do extrato fitoterápico (uma vez a cada

Tabela I. Valores médios e coeficiente de variação (CV) do tempo de cicatrização e queda do coto umbilical de cabritos tratados com diferentes tinturas fitoterápicas (Average values and coefficient of variation (CV) of the healing time and fall of the umbilical stump of kid goats treated with different herbal tinctures).

\begin{tabular}{ccccc}
\hline & \multicolumn{3}{c}{ Tratamentos } & \\
\cline { 2 - 4 } Item & Tintura & Tintura de & Tintura de & CV (\%) \\
de lodo & Aroeira & Babosa \\
& $(\mathrm{GC})$ & $(\mathrm{G} 1)$ & $(\mathrm{G} 2)$ & \\
\hline
\end{tabular}

\section{Tempo de}

cicatrização

$20,4^{a}$

$20,8^{a}$

$18,6^{\mathrm{a}}$

(dias)

Médias com letras iguais na mesma linha não diferem pelo teste de Tukey $(P<0,05)$.
12 horas até a completa cicatrização e queda do coto umbilical) não promoveu rapidez no processo cicatricial. Pode-se inferir que o maior tempo de cicatrização ocorreu em virtude do excesso de hidratação dos tecidos do coto umbilical. Adicionalmente, este resultado reforça a inviabilidade desse método, por aumentar a necessidade de mão-de-obra, gerar maior custo decorrente da aquisição de produtos e plantas medicinais e, além disso, tornar o coto umbilical susceptível à infecção por agentes patogênicos por maior número de dias.

Por outro lado, nos animais submetidos aos tratamentos com apenas uma aplicação diária por três dias consecutivos (ensaio II), notou-se que o tempo médio para cicatrização e queda do coto umbilical não diferiu $(\mathrm{P}>0,05)$ entre os tratamentos avaliados, de modo que estes eventos aconteceram em aproximadamente 20 dias (Tabela I), divergindo do preconizado pela ACCOMIG (n.d.).

Vale ressaltar que o maior tempo para cicatrização e queda do coto umbilical encontrado para os animais do GC do ensaio II em relação ao ensaio I (20 vs. 11 dias, respectivamente), ambos com utilização de tintura de iodo a $10 \%$ por três dias seguidos, pode ter ocorrido devido ao fato dos ensaios experimentais terem sido realizados em diferentes anos e estações, com condições ambientais distintas (umidade relativa do ar e precipitação pluviométrica, por exemplo). Em adição, no ensaio II a solução iodada foi aplicada uma única vez ao dia, o que pode não ter sido suficiente para que ocorresse rápida cicatrização dos tecidos umbilicais, como pode ser percebido nos animais do GC do ensaio I.

A partir da análise dos custos de produção verificou-se a viabilidade econômica das tinturas fitoterápicas utilizadas no estudo (Tabela II), devido ao menor

Tabela II. Avaliação econômica do uso de tinturas fitoterápicas no tratamento do coto umbilical de cabritos (Economic evaluation of the use of herbal tinctures to treat the umbilical stump of kid goats).

\begin{tabular}{|c|c|c|c|c|}
\hline Tratamento & $\begin{array}{l}\text { Número de } \\
\text { animais }\end{array}$ & $\begin{array}{l}\text { Quantidade } \\
\text { do produto } \\
\text { (L) }\end{array}$ & $\begin{array}{l}\text { Valor do } \\
\text { produto } \\
(\mathrm{R} \$ / L)\end{array}$ & $\begin{array}{l}\text { Custo } \\
(\mathrm{R} \$)^{*}\end{array}$ \\
\hline $\begin{array}{l}\text { Tintura de } \\
\text { lodo }(\mathrm{GC})^{\star *}\end{array}$ & 7 & 1,05 & 60,00 & 63,00 \\
\hline $\begin{array}{l}\text { Tintura de } \\
\text { lodo }(\mathrm{G} 1)^{\star *}\end{array}$ & 7 & 10,85 & 60,00 & 651,00 \\
\hline $\begin{array}{l}\text { Tintura de } \\
\text { Aroeira } \\
(\mathrm{G} 2)^{\star \star}\end{array}$ & 7 & 14,00 & 5,00 & 70,00 \\
\hline $\begin{array}{l}\text { Extrato de } \\
\text { Babosa } \\
(\mathrm{G} 3)^{\star *}\end{array}$ & 7 & - & - & - \\
\hline $\begin{array}{l}\text { Tintura de } \\
\text { lodo*** }\end{array}$ & 5 & 0,375 & 60,00 & 22,50 \\
\hline $\begin{array}{l}\text { Tintura de } \\
\text { Aroeira**** }\end{array}$ & 5 & 0,375 & 5,00 & 1,90 \\
\hline $\begin{array}{l}\text { Tintura de } \\
\text { Babosa }{ }^{\star * \star}\end{array}$ & 5 & 0,375 & 6,00 & 2,25 \\
\hline
\end{tabular}

Archivos de zootecnia vol. 67, núm. 258, p. 286. 
custo decorrente do uso de plantas medicinais em detrimento do iodo, que atualmente no comércio pode ser adquirido por um valor médio de R\$ 60,00/litro, enquanto que a aroeira e a babosa encontram-se abundantemente disponíveis em várias regiões do Brasil. Sendo assim, os custos para a elaboração das tinturas fitoterápicas se concentram apenas na aquisição dos solventes, o que implica em maior vantagem econômica quando comparado ao uso de tintura de iodo.

É relevante salientar que não foi constatada incidência de infecções umbilicais em nenhum animal dos grupos experimentais, tanto no ensaio preliminar quanto no experimento de campo (II). Nesse contexto, os resultados encontrados sinalizam os fitoterápicos empregados como uma importante ferramenta de contribuição para melhoria do manejo de crias na caprinocultura, tendo em vista que auxiliam os produtores rurais na adoção do tratamento do coto umbilical de maneira pouco onerosa e com resultados semelhantes aos encontrados para a utilização de solução iodada.

\section{CONCLUSÕES}

As tinturas fitoterápicas à base de aroeira ou babosa, aplicadas uma vez por dia durante três dias consecutivos, podem substituir a tintura de iodo a $10 \%$ e demonstram maior viabilidade econômica para utilização no processo de cicatrização e queda do coto umbilical de cabritos.

\section{BIBLIOGRAFIA}

Amaro, LPA, Maciel, MV, Lucena, JA, Lima Júnior, DM, \& Sombra, DS 2011, 'Utilização do extrato aquoso da babosa (Aloe vera) no manejo higiênico de ordenha em cabras', ACSA - Agropecuária Científica no Semi-Árido, vol. 7, no. 1, pp. 06-10.
Castelo Branco Neto, ML, Ribas Filho, JM, Malafaia, O, Oliveira Filho, MA, Czeczko, NG, Aoki, S, Cunha, R, Fonseca, VR, Teixeira, HM, \& Aguiar, LRF 2006, 'Avaliação do extrato hidroalcoólico de Aroeira (Shinus terebinthifolius Raddi) no processo de cicatrização de feridas em pele de ratos', Acta Cirúrgica Brasileira, vol. 21, pp. 17-22.

Catalan, AAS, Gopinger, E, Lopes, DCN, Gonçalves, FM, Roll, AAP, Xavier, EG, Avila, VS, \& Roll, VFB 2012, 'Aditivos fitogênicos na nutrição animal: Panax ginseng', Revista Portuguesa de Ciências Veterinárias, vol. 107, no. 581-582, pp. 15-21.

Associação dos Criadores de Caprinos e Ovinos do Estado de Minas Gerais n.d., A cura do cordão umbilical, ACCOMIG, Belo Horizonte, acessado 21 mar. 2015, http://www.caprileite.com.br/conteudo/31II-a-cura-do-cordao-umbilical

Maia-Filho, ALM, Silva,VS, Barros, TL, \& Costa, CLS 2011, 'Efeito do gel da babosa (Aloe barbadensis Mill.) associado ao ultrassom em processo inflamatório agudo', Revista Brasileira de Plantas Medicinais, vol. 13, no. 2, pp. 146-50.

Medeiros, JM, Tabosa, IM, Simões, SVD, Nóbrega Júnior, JE, Vasconcelos, JS, \& Riet-Correa, F 2005, 'Mortalidade perinatal em cabritos no semi-árido da Paraíba', Pesquisa Agropecuária Brasileira, vol. 25, no. 4, pp. 201-06.

Mendonça, VM, Santos, AJ, Nascimento, IR, Oliveira, MAS, Rocha, SS, \& Cabral, ES 2014, 'Perspectivas da fitoterapia veterinária: plantas potenciais na terapia dos animais de produção', Cadernos de Agroecologia, vol. 9, no. 4, pp. 1-5.

Moneam, NMA \& Ghoneim, T 1986, 'Gas chromatographic analysis of total 1 fatty acids extracted from $S$. terebinthifolius berries', Journal of Chromatography A, vol. 361, pp. 391-95.

Oliveira, LST, Silva, SLC, Tavares, DC, Santos, AV, \& Oliveira, GCB 2009, 'Uso de plantas medicinais no tratamento de animais', Centro Científico Conhecer - Enciclopédia Biosfera, vol. 5, no. 8, pp. 1-8. Olivo, CJ, Carvalho, NM, Silva, JHS, Vogel, FF, Massariol, P, Meinerz, G, Agnolin, C, Morel, AF, \& Viau, LV 2008, 'Óleo de citronela no controle do carrapato de bovinos', Ciência Rural, vol. 38, no. 2, pp. 406-10. 